\title{
A Short Note on a Nonlinear System Vibrations under Two Non-Ideal Excitations
}

Universidade Regional Integrada de Santo Ângelo Departamento de Ciências Exatas e da Terra-URI

C.P. 203

98802-470 Santo Ângelo, RS. Brazil jfelix@urisan.tche.br

\author{
J. M. Balthazar \\ Universidade Estadual Paulista \\ Instituto de Geociências e Ciências Exatas \\ Departamento de Estatística, Matemática Aplicada \\ e Computação - UNESP \\ C.P. 178 \\ 13500-230 Rio Claro, SP. Brazil \\ jmbaltha@rc.unesp.br \\ R. M. L. R. F. Brasil \\ Universidade de São Paulo, Escola Politécnica \\ Departamento de Estrutura e Fundações - USP \\ C. P. 61546 \\ 9524-970 São Paulo, SP. Brazil \\ rmlrdfbr@usp.br
}

This paper describes a nonlinear phenomenon in the dynamical behavior of a nonlinear system under two non-ideal excitations: the self-synchronization of unbalanced direct current motors. The considered model is taken as a Duffing system that is excited by two unbalanced direct current motors with limited power supplies. The results obtained by using numerical simulations are discussed in details

Keywords: Nonlinear system, non-ideal system, self-synchronization

\section{Introduction}

We remark that the study of non-ideal vibrating systems, that is, those where the excitation is influenced by the response of the system, is still considered to be a major challenge in theoretical and practical engineering research.

When the excitation is not influenced by the response, it is said to be an ideal excitation or an ideal source of energy. On the other hand, when the excitation is influenced by the response of the system, it is said to be non-ideal. Thus, depending on the excitation, one refers to vibrating systems as ideal or non-ideal.

The behavior of ideal vibrating systems is well known in current literature, but there are few published results on non-ideal ones. Generally, non-ideal vibrating systems are those for which the power supply is limited. The behavior of the vibrating systems departs from the ideal case as power supply becomes more limited. For non-ideal dynamical systems, one must add an equation that describes how the energy source supplies the energy to the equations that govern the corresponding ideal dynamical system. Thus, as a first characteristic, the non-ideal vibrating system has one more degree of freedom than its ideal counterpart.

The first kind of non-ideal problem arising in current literature is the so-called Sommerfeld effect, discovered in 1902 (see Sommerfeld, 1902), discussed in a book by (Kononenko, 1969), and entirely devoted to the subject. Recently, a review of different theories concerning this subject, was presented in (Balthazar et al, 2001), (Balthazar et al., 2002) and (Palacios, 2002).

Self-synchronization of shafts is a well-known nonlinear phenomenon, whereby two (or more) unbalanced shafts on a common movable structure may rotate synchronously due to interaction via structural vibrations only, even in the absence of any direct kinematics coupling. The phenomenon has been extensively

Paper accepted October, 2003. Technical Editor: Atila P. Silva Freire. studied by asymptotic methods to predict possible (multiple) steadystate rotational motions and to evaluate their stability, mostly with application to the design of vibrators with a reduced number of driving motors. Certain cases of undesirable shaft selfsynchronization in engineering have also been studied, but only steady-state motions were analyzed. Results of numerical simulation of transient self-synchronization of rotating shafts, one potential application being gas turbine engines with multiple shafts, was studied by (Dimentberg, 2001).

In this paper, two unbalanced de motors are used to demonstrate the self-synchronization that may occur when the shafts rotation speeds become temporarily close to one another depending on the torque, considered as the control variable, and of a support with nonlinear stiffness.

This paper is an extension of the following previous works: (Palacios, 2002) that studied a portal frame with nonlinear characteristic of elasticity under one non-ideal excitation; (Balthazar et al., 2001) and (Warminski and Balthazar, 2001) and (Warminsky and Balthazar, 2003) that studied the non-stationary regime of a DC motor with limited power supply; (Kang, 2002) that studied the non-linear dynamic of non-linear systems subjected to double excitations; (Dimentberg et al., 2001) that studied the selfsynchronization of rotors and Sommerfeld effect and (Blekhman, 1988) that studied the self-synchronization of two unbalanced rotating machines mounted on a linearly elastic support. A first announcement of this work was done by (Palacios et al, 2003).

\footnotetext{
Nomenclature

$\hat{a}=$ control parameter, dimensionless

$\hat{b}=$ parameter related to a type of motor, dimensionless

$F_{d}=$ Non-linear damping function

$F_{s}=$ Non-linear stiffening function

$J_{l}=$ moment of inertia of rotor $1, \mathrm{kgm}^{2}$

$\mathrm{J}_{2}=$ inertia moment of rotor $2, \mathrm{Kgm}^{2}$

$k_{c}=$ friction coefficient in the bearing
} 
$k_{M}=$ motor parameter

$M_{1}=$ driving torque of the motor $1, \mathrm{Nm}$

$M_{2}=$ driving torque of the motor $2, \mathrm{Nm}$

$m_{0}=$ total mass of vibrating parts, $\mathrm{kg}$

$x=$ generalized coordinate, dimensionless

\section{Greek Symbols}

$\varphi_{1}=$ angular displacement of motor $1, \mathrm{rad}$.

$\varphi_{2}=$ angular displacement of motor $2, \mathrm{rad}$.

$\hat{\omega}=$ natural frequency of the system, dimensionless

\section{Subscripts}

$1=$ relative to the horizontal displacement

$2=$ relative to the vertical displacement

\section{Dynamical Model of the Nonlinear System}

Consider a nonlinear mechanical system consisting of two unbalanced rotors driven by two dc motors with limited power supplies and mounted on an elastic support with nonlinear stiffening and damping. Figure 1 illustrates such a system.

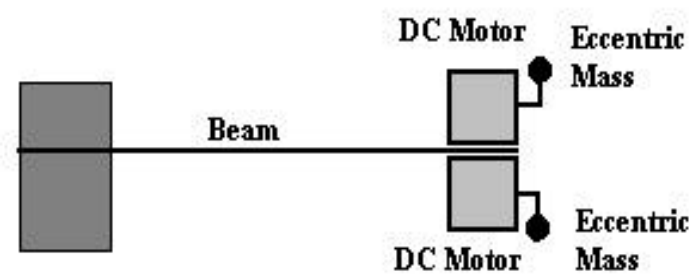

Figure 1. Schematic representation of the cantilever beam supporting two non-ideal motors.

The differential equations of motion may be written as follows (see, for instance, Nóbrega, 1994; Dimentberg et al., 2001; Warminski and Balthazar, 2001; Balthazar et al., 2001; Kang et al., 2002; Blekhman, 1988 and Palacios, 2002):

$$
\begin{gathered}
m_{0} \ddot{x}+F_{d}(x)+F_{s}(x)=q_{1}\left(\ddot{\varphi}_{1} \sin \varphi_{1}+\varphi_{1}^{2} \cos \varphi_{1}\right) \\
+q_{2}\left(\ddot{\varphi}_{2} \sin \varphi_{2}+\varphi_{2}^{2} \cos \varphi_{2}\right) \\
J_{1} \ddot{\varphi}_{1}=\alpha_{1} \ddot{x} \sin \varphi_{1}-\alpha_{2} \cos \varphi_{1}+k_{M} M_{1}(t)-k_{c} \dot{\varphi}_{1} \\
J_{2} \ddot{\varphi}_{2}=\alpha_{3} \ddot{x} \sin \varphi_{2}-\alpha_{4} \cos \varphi_{2}+k_{M} M_{2}(t)-k_{c} \dot{\varphi}_{2},
\end{gathered}
$$

where

$m_{0}=$ is the total mass of vibrating parts,

$J_{1}, J_{2}=$ moment of inertia of motors 1 and 2,

$F_{d}(\dot{x})=$ Non-linear damping function (for Van der Pol model, it is

$\left.\gamma\left(x^{2}-1\right) \dot{x}\right)$,

$F_{S}(x)=$ Non-linear stiffening function (for Duffing model, it is

$\left.k_{1} x+k_{2} x^{3}\right)$,

$\varphi_{1}, \varphi_{2}=$ Rotation angles of the rotors measured from the lowest vertical position,

$x=$ Vertical displacement of the supporting body from the

equilibrium position,

$k_{c}=$ Friction coefficient of bearings,

$k_{M}=$ Motor parameter,

$M_{1}, M_{2}=$ The torques of the motors, $q_{1}, q_{2}, \alpha_{j}(j=1 \ldots 4)=$ Parameters depending on the eccentricities of rotors and physical parameters of the system.

The motor torque can be expressed (Balthazar et al., 2001), for non-stationary regime, by the following expressions:

$$
\begin{gathered}
\dot{M}_{1}=-p_{1} M_{1}-p_{2} \dot{\varphi}_{1}+k_{U} U_{1} \\
\dot{M}_{2}=-p_{3} M_{2}-p_{4} \dot{\varphi}_{2}+k_{U} U_{2}
\end{gathered}
$$

and, if stationary regime is considered, the above expressions become:

$$
\begin{aligned}
& M_{1}=\frac{k_{U}}{p_{1}} U_{1}-\frac{p_{2}}{p_{1}} \dot{\varphi}_{1} \\
& M_{2}=\frac{k_{U}}{p_{3}} U_{2}-\frac{p_{4}}{p_{3}} \dot{\varphi}_{2}
\end{aligned}
$$

where $U_{1}, U_{2}$ are voltages applied across the armature of the motors.

Next, we will consider the non-ideal Duffing model in dimensionless form:

$$
\begin{aligned}
& x^{\prime \prime}+\hat{\mu} x^{\prime}+\hat{\omega}^{2} x+\hat{p} x^{3}= \hat{q}_{1}\left(\varphi_{1}^{\prime \prime} \sin \varphi_{1}+\varphi_{1}^{\prime 2} \cos \varphi_{1}\right) \\
&+\hat{q}_{2}\left(\varphi_{2}^{\prime \prime} \sin \varphi_{2}+\varphi_{2}^{\prime 2} \cos \varphi_{2}\right) \\
& \varphi_{1}^{\prime \prime}=\hat{\alpha}_{1} x^{\prime \prime} \sin \varphi_{1}-\hat{\alpha}_{2} \cos \varphi_{1}+\hat{a}_{1}-\hat{b} \varphi_{1}^{\prime} \\
& \varphi_{2}^{\prime \prime}=\hat{\alpha}_{3} x^{\prime \prime} \sin \varphi_{2}-\hat{\alpha}_{4} \cos \varphi_{2}+\hat{a}_{2}-\hat{b} \varphi_{2}^{\prime} .
\end{aligned}
$$

where

$\tau=\omega t$ Defined as dimensionless time,

$\hat{a}_{1}, \hat{a}_{2}=$ Dimensionless torques applied by the dc motors, used as control parameters,

$\hat{b}=$ identical dc motors model,

$\hat{\mu}=$ dimensionless damping coefficient,

$\hat{\omega}=$ dimensionless natural frequency of the Duffing oscillator,

$\hat{p}=$ dimensionless cubic stiffness coefficient.

In the next section we will study the self-synchronization of the non-ideal system represented by differential equations (4) using the characteristic curve of the motors in the straight-line approximation (3).

\section{Numerical Simulation Results}

To simulate the mathematical model of the system (4), MATLAB-SIMILUNK® Software, was used. Differential equations were solved by integration method of Runge-Kutta. Numerical, dimensionless data for the non-ideal non-linear system are shown in Table 1.

Table 1. Systems parameters.

\begin{tabular}{cl}
\hline Dynamical System & \multicolumn{1}{c}{$\begin{array}{c}\text { Numerical value of the } \\
\text { dimensionless parameters }\end{array}$} \\
\hline & $\hat{\mu}=0.1, \hat{\omega}=1, \hat{p}=0.1$, \\
Non-ideal Duffing & $\hat{q}_{1}=0.2, \hat{q}_{2}=0.2$ \\
equation (3) and (4) & $\hat{\alpha}_{1}=\hat{\alpha}_{3}=0.3, \hat{\alpha}_{2}=\hat{\alpha}_{4}=0$, \\
& $\hat{b}=1.5$ \\
\hline
\end{tabular}


Results of computations are presented Fig. 2-4. Behavior of the system was observed during the selection of the torques as $\hat{a}_{1} \approx \hat{a}_{2}$, $\hat{a}_{1} \neq \hat{a}_{2}$.

We analyze the self-synchronization of the two unbalanced rotors in post-resonance and resonance regions related to the difference between rotors velocities. Figures 2(a) and 4 (a) have an expanded portion bellow.

\section{First Set of Numerical Simulation Results:}

Figure 2 illustrates the development of self-synchronization in the non-ideal and non-linear mechanical system when the dimensionless torques are $\hat{a}_{1}=5.0, \hat{a}_{2}=5.04$. We observe that the rotors turn in the same direction and arrive to some average rotational velocity in steady state motion, see Fig. 2(a), where the rotational velocities are in anti-phase (the rotors synchronize antiphase), see the zoom of Fig. 2(a) bellow. The velocities of the rotors are out of the resonance region. Note also that the rotor velocity (difference) approaches zero in the stationary regime, see Fig 2(c). At the same time, the vertical displacement of the supporting body decreases; see Fig. 2(b).
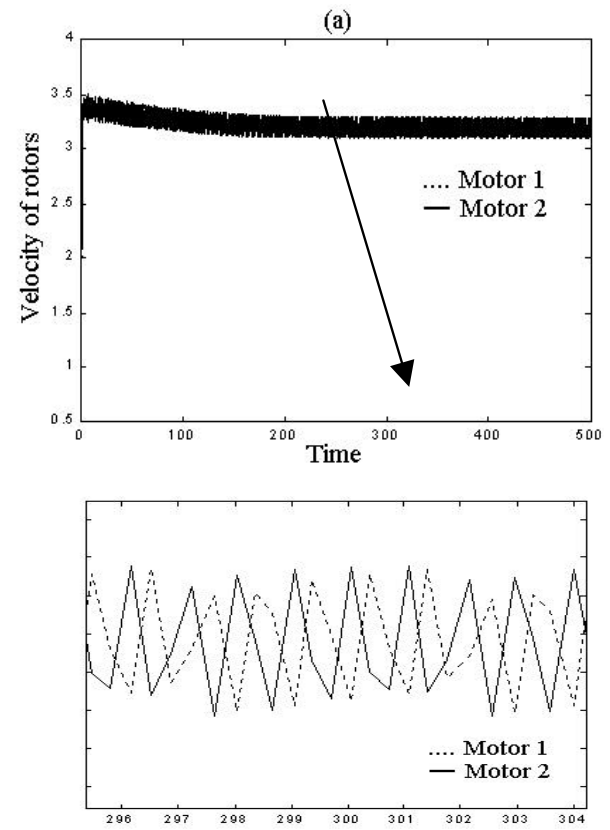

(b)

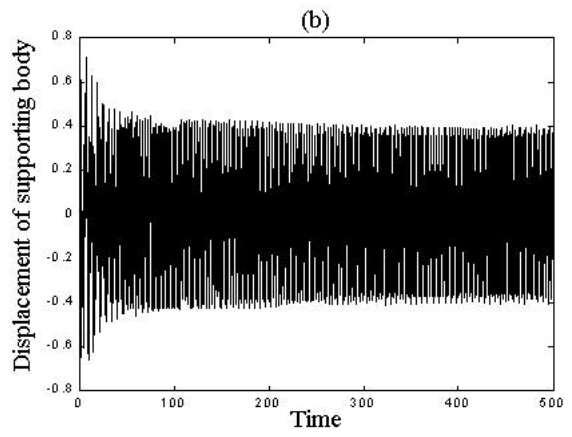

Figure 2. Self-synchronization of the unbalanced rotors with torques $\hat{a}_{1}=5.0, \hat{a}_{2}=5.04$.

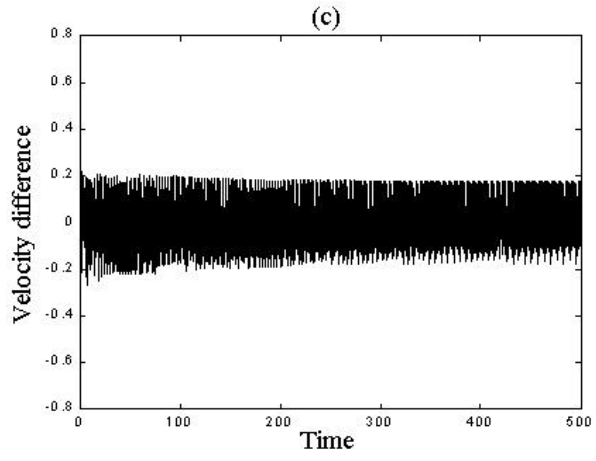

Figure 2. (Continued).

\section{Second Set of Numerical Simulation Results:}

Figure 3 illustrates the absence of self-synchronization when the torques are different, $\hat{a}_{1}=5.0$ and $\hat{a}_{2}=1.5$. It is seen that the rotational velocities of the rotors have different values.

The rotational velocity of the second rotor is captured at the resonance region of the system and the rotational velocity of the first rotor is above of the resonance region (see Fig. 3(a)). Note that the vertical displacement of the supporting body does not decrease (see Fig. 3(c)), and note also that the rotor velocity difference (see. Fig. 3(b)) does not tend to zero; i.e. the self-synchronization is absent.

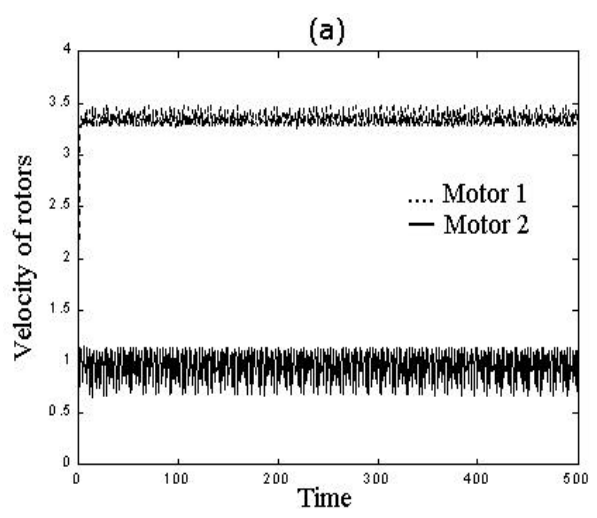

(b)

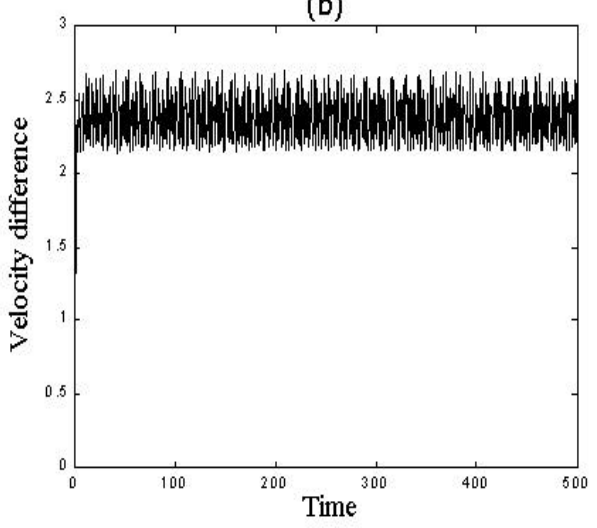

Figure 3. Absence of self-synchronization of the unbalanced rotors with torques $\hat{a}_{1}=5.0$ and $\hat{a}_{2}=1.5$. 
(c)

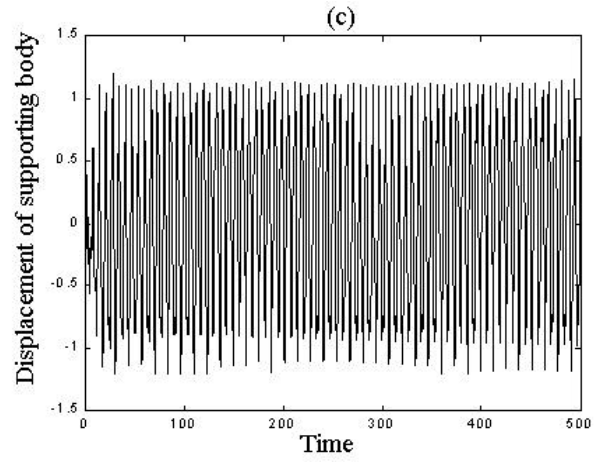

Figure 3. (Continued).

\section{Third Set of Numerical Simulation Results:}

Figure 4 shows the synchronization for a certain time interval, where we may observe the reduction of the damping of the support. We also observe that the response follows the some synchronization of the rotation of the rotors. In this case the constant torques considered were $\hat{a}_{1}=1.5$ and $\hat{a}_{2}=3.0$.

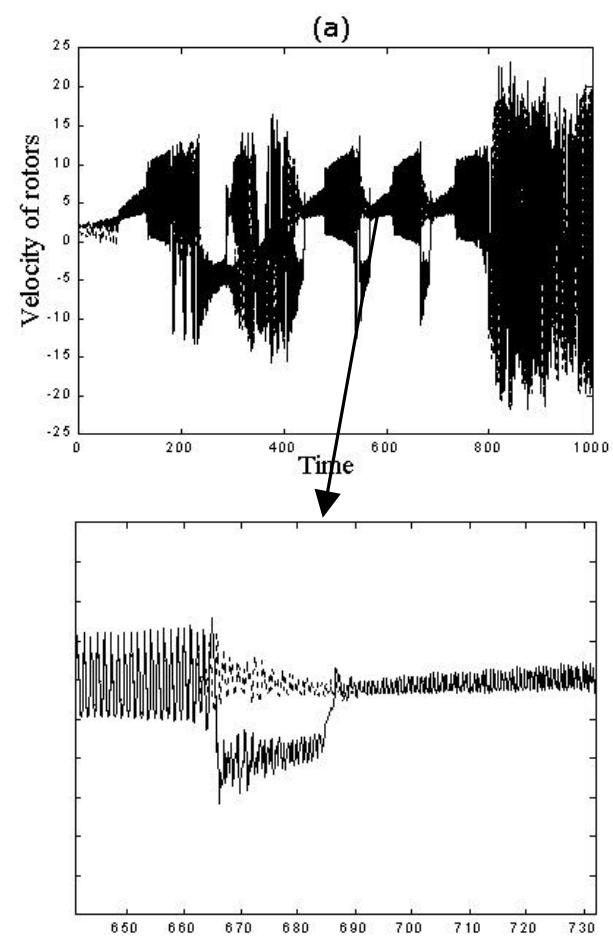

Figure. 4. Simulations results when we reduce the damping of the supporting $\hat{\mu}=0.001$ with $\hat{a}_{1}=1.5$ and $\hat{a}_{2}=3.0$.

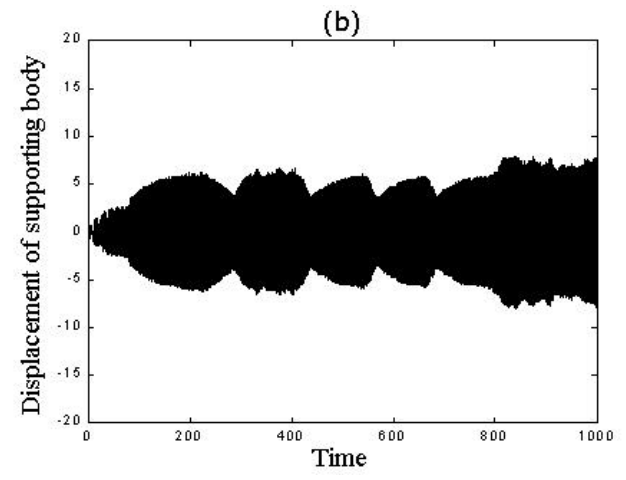

(c)

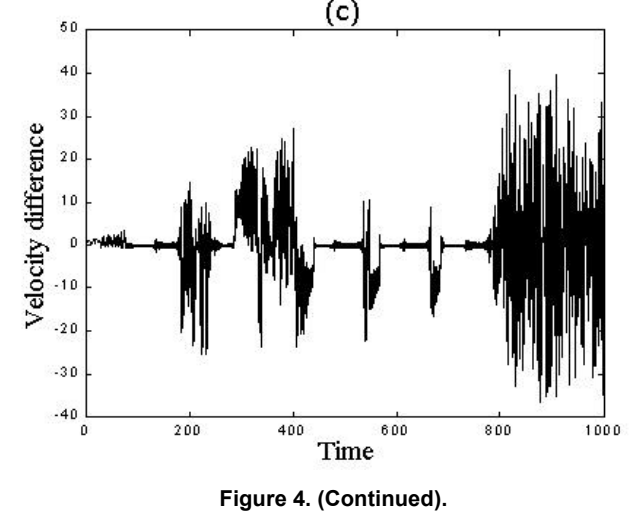

On other hand, we observe chaotic behavior in the interaction between the Duffing oscillator and the two unbalanced rotors when damping of the support is reduced (see Figs 4(b) and (c)).

\section{Conclusions}

A practical problem of synchronization of a non-ideal and nonlinear vibrating system was posed and investigated by means of numerical simulations. It has also been shown, that by making constant the variation of torques, we may control the selfsynchronization and synchronization (in the system).

This work has as its motivation the investigation of a class of vibrating machines: crushers, mills, screens, feeder, etc. We developed mathematical models and implemented numerical analysis that may also be used for control purposes.

\section{Acknowledgements}

The first author thanks to the department of Exacts Sciences of URISAN that provided computational material support and the second and third authors thank grants by FAPESP and CNPq, both Brazilian Research Funding Agencies

\section{References}

Balthazar, J.M., Mook, D.T., Weber H.I., Brasil, R.M.L.R.F., Fenili, A., Belato, D., Felix, J.L.P., 2003, "An overview on non-ideal vibrations", Meccanica, in press.

Balthazar, J.M., Mook, D.T., Weber H.I., Brasil, R.M.L.R.F., Fenili, A., Belato, D., Felix, J.L.P., 2001, "Recent Results on Vibrating Problems with Limited Power Supply", In: Sixth Conference on dynamical Systems Theory and Applications, Lodz, Poland, December, 10-12, pp. 27-50.

Balthazar, J.M., Cheshankov, B.I., Ruchev, D.T., Barbanti, L., Weber, H.I., 2001, "Remarks on the Passage Through Resonance of a Vibrating 
System with Two Degrees of Freedom, Excited by a Non-Ideal Energy Source", Journal of Sound and Vibration, 239(5), pp. 1075-1085. Blekhman, I.I., 1988, "Self-Synchronization in Science and Technology", ASME Press, New York.

Blekhman, I.I., 2000, "Vibrational Mechanics: Nonlinear Dynamic Effects, General Approach, Applications", World Scientific.

Dimentberg, M., Cobb, E. and Mensching, J., 2001, "SelfSynchronization of Transient Rotations in Multiple Shaft Systems", Journal of Vibration and Control, 7, pp. 221-232.

Kang, Y., Chao, C.P., Chou, C.C., Chu, M.H., Mu, L.H., 2002, "An Integration Method for Detecting Chaotic Responses of Nonlinear Systems Subjected to Double Excitations", International Journal of Non-Linear Mechanics, 37, pp. 1073-1077.

Kononenko, V., 1969, "Vibrating Systems with Limited Power Supply", Illife.

Nóbrega, P.G.B., 1994, “Auto-sincronização de Motores Não-ideais Apoiados em Estruturas Elasticas”, Dissertação de Mestrado, EP, Universidade de São Paulo.
Palacios, J.L., 2002, "Teoria de Sistemas Vibratórios Não-lineares e Não-ideais", Tese de Doutorado, Universidade Estadual de Campinas, Campinas, SP, Brasil, 181p.

Palacios, J.L. Balthazar J.M., Brasil, R.M.L.R.F, 2002, “On Non-ideal and Non-linear Portal Frame Dynamic Analysis Using Bogoliubov Perturbation Method", Journal of the Brazilian Society of Mechanical Systems, Vol XXIV, $\mathrm{N}^{\circ}$ 4, pp. 257-265.

Palacios, J.L., Balthazar J.M., Brasil, R.M.L.R.F, 2003, “ On the NonLinear Dynamics of a Non-Linear System under Two Non-ideals Excitations", In: Proceedings of the X DINAME, 10-14 Th. March 2003, Ubatuba, SP, Brazil, and pp. 381-386.

Sommerfeld, A., 1902, "Beiträge Zum Dynamischen Ausbau Der Festigkeitslehe", Physikal Zeitschr, (3), p. 266-286.

Warminski, J. and Balthazar, J.M., 2001, "On Interactions between Parametric and Self-Excited Vibrations on the System with Ideal and Nonideal Source", In: Sixth Conference on dynamical Systems Theory and Applications, Lodz, Poland, December 10-12, pp. 407-414. 